\title{
Design of a Linear-Assisted VLC-LED Transmitter Based on Summing the Light
}

\author{
Daniel G. Aller, Diego G. Lamar, Pablo F. Miaja, Juan Rodríguez y Javier Sebastián. \\ \{garciaadaniel, gonzalezdiego, fernandezmiapablo, rodriguezmjuan, sebas\}@ uniovi.es \\ Electrical, Electronic, Computers and Systems Engineering Department, \\ University of Oviedo, Gijón 33204, Spain.
}

\begin{abstract}
:
A high efficiency linear-assisted Visible Light Communication (VLC) LED driver working as a transmitter is presented in this work. A linear-assisted transmitter is based on the idea of using a high efficiency but bandwidth limited circuitry which delivers most of the power and a low efficiency but fast linear amplifier (linear assistance) which corrects the distortion of the signal. The presented solution takes advantage of the light in a VLC system by summing the contribution of the linear amplifier in light instead of electrically, which leads to a electrical isolation between both stages and a reduction of the complexity comparing to the traditional approach used in Envelope Tracking (ET) and Envelope Elimination and Restoration (EER) techniques. As experimental results, the design of a linear-assisted Class $\mathrm{E}$ amplifier delivering a 16-PSK phase digital modulation with a $1 \mathrm{MHz}$ carrier is presented. On one hand, the Class $\mathrm{E}$ amplifier delivers most of the power (92\% of the signal power) at high efficiency (81\%) and, on the other hand, the linear amplifier only delivers the error signal at lower efficiency. The efficiency of the signal circuitry is up to $75 \%$ and the efficiency of the whole system considering the signal and the LED biasing circuitry is up to $85 \%$.
\end{abstract}

\section{INTRODUCTION}

In recent years, the wireless traffic has been increasing dramatically which is based almost entirely on the Radio Frequency (RF) spectrum. The current situation and the predicted increase over the next few years will lead to a congestion and a more stricted regulation of the RF spectrum [1].

One of the most promising alternatives to avoid the congestion of the RF spectrum is Visible Light Communication (VLC) [2], [3]. On one hand, VLC takes advantage of the wide and unregulated visible light spectrum $(280 \mathrm{~nm}-750$ $\mathrm{nm}$ ) and, on the other hand, VLC uses the widespread SolidState Lighting (SSL) based on LED. Due to the fast light modulation capability of the LED, it is able to perform fast variations of the emitted light, making it suitable as emitter for VLC. VLC as been proposed as a promising alternative

This work was supported in part by European Regional Development Fund grants, in part by the Spanish Government under project MINECO-17DPI2016-75760-R, and in part by the Principality of Asturias under project IDI/2018/000179 and scholarship BP17-91. for application such as indoor positioning, vehicle to vehicle communication, wireless sensor networks, etc. [4], [5].

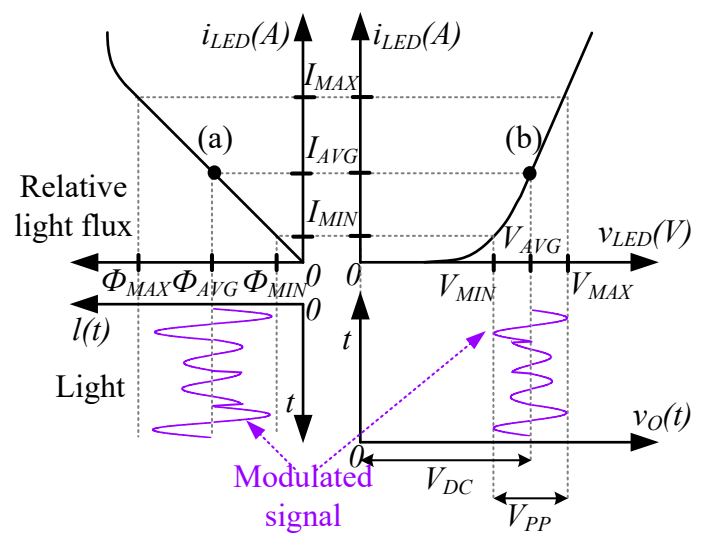

Figure 1: The current vs voltage and light vs current in a LED working as VLC emitter.

Due to the use of the SSL infrastructure, a VLC system is meant to perform the lightning and the communication task at the same time. Figure 1 shows the well-known current vs voltage and light vs current characteristics in a LED. In order to illustrate both tasks, a modulated voltage signal is applied. The average value of the signal $V_{A V G}$ biases the LED, leading to an average current $I_{A V G}$ and a average emitted light $\Phi_{A V G}$. Providing that the communication signal is fast enough to be unnoticeable by the human eye, the average value would control the illumination task. Around the average value, the communication signal is applied and as long as the LED is working in its linear region, the current and the light would be proportional to the applied communication signal.

Since there are two different tasks and these can be performed by different circuitry, two different efficiencies are taken into account: the efficiency of the circuitry that generates the signal and the efficiency of the LED biasing circuitry.

One of the main challenges in VLC is to provide a high bit rate communication without deteriorate excessively the high electrical efficiency of the overall SSL system. One of the solutions that has been proposed is the use of a linear amplifier stage performing the communication task (i.e. Class A, B or $\mathrm{AB})$ [6]-[9] which leads to a high bit rate performance but with a low power efficiency in the circuitry that generates the signal. Other solution is the use of DC-DC converter 
and switching mode amplifiers (i.e. Class D or E) as VLC transmitters which improves the power efficiency [10]-[13] but with a limited bandwidth, slow slew-rate and more complex control.

In the later solution, the limited bandwidth and low slewrate compromises the communication performance when a fast response is required by the communication signal. In order to keep a high efficiency and at the same time to have a faster response when necessary, the use of a linear amplifier stage in parallel has been widely use, especially in Envelope Tracking (ET) and Envelope Elimination and Restoration (EER) techniques [14]-[16]. A trade-off between slew-rate and efficiency must be establish due to the low efficiency of the linear stage. The higher the slew-rate, the higher the power delivered by the linear amplifier stage so the lower the overall efficiency.

In this paper, an adaptation of the linear-assisted technique for VLC is presented. The transmitter is based on a Class E amplifier assisted by a linear amplifier. The Class E amplifier generates the digital phase modulation, delivering most of the power of the communication stage ( $92 \%$ of the signal power) at high efficiency $(81 \%)$ and the linear amplifier stage only delivers power in those moments when the Class E amplifier does not meet the bandwidth and slew-rate required by the modulation (i.e. in a phase step). The technique is adapted to VLC and by taking advantage of the light. Both signals are added in their light form, so the parallel connection between the two amplifiers is not longer necessary. Adding the signals in light intensity provides electrical isolation between both amplifiers which avoids the undesirable influence between them. As experimental results a 16-PSK digital phase modulation is used with a $1 \mathrm{MHz}$ carrier, which achieves a bit-rate up to $0.5 \mathrm{Mbps}$ and a distance up to $1 \mathrm{~m}$. The signal efficiency, considering the Class $\mathrm{E}$ amplifier and the linear amplifier contributions is $75 \%$ and the overall efficiency, considering signal and biasing is up to $85 \%$.

\section{PRINCIPLE OF OPERATION OF A LinEAR-ASSISTED TRANSMITTER}

Figure 2 shows the block diagram and figure 3 an example of the waveforms of a linear assisted DC-DC converter used typically in ET [17] and EER [18] techniques. The main idea behind ET and EER is varying the power supply voltage of the Radio Frequency Power Amplifier (RFPA) according to the envelope of the communication signal $s_{c o m m}(t)$, which increases considerably the efficiency of the RFPA. In order to do this, an external DC-DC converter is meant to deliver the signal $s_{i n}(t)$ to the RFPA. The technique takes advantage of the high efficiency of the DC-DC converter but because of the limited bandwidth and slow slew rate, the signal that is being delivered is $s_{D C-D C}(t)$, close to the $s_{i n}(t)$ but with noticeable distortion. In order to reduce the distortion, a much faster and with higher bandwidth circuitry is used, such as an auxiliary linear amplifier. The linear amplifier gives the linearassistance by delivering the error signal $s_{\text {err }}(t)$ between $s_{i n}(t)$ and $s_{D C-D C}(t) \cdot s_{D C-D C}(t)$ and $s_{e r r}(t)$ are combined and

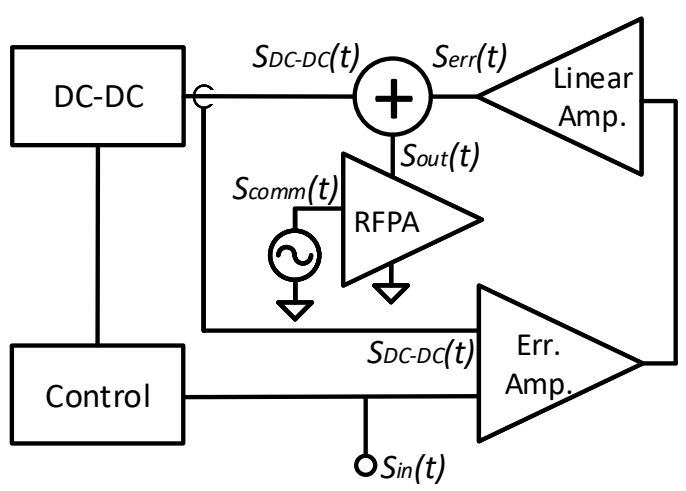

Figure 2: Block diagram of a linear-assisted DC-DC converter used in ET and EER.

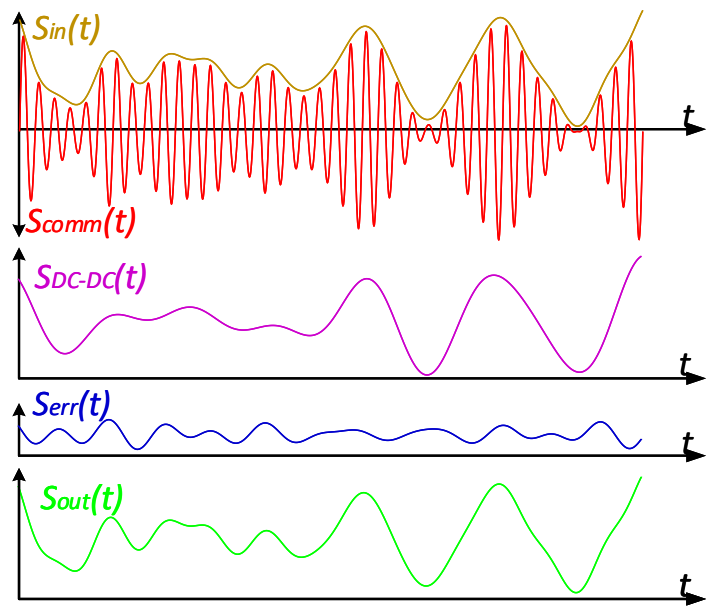

Figure 3: Working example of the main waveforms in a linear-assisted transmitter.

used as the power supply voltage for the main RFPA. The lower the difference between the amplitude of $s_{\text {comm }}(t)$ and $s_{\text {out }}(t)$, the higher the efficiency of the RFPA. One of the main challenges of this technique is the design of the combiner that connects both circuits, controlling both the power flow and the contribution of both circuits in order to keep a high efficiency.

For high efficiency performance on the power supply side (DC-DC converter + the linear-assistance), the DC-DC converter must deliver most of the power, so a trade-off between slew-rate and efficiency must be establish due to the low efficiency of the linear amplifier stage. The higher the slewrate, the higher the power delivered by the linear amplifier stage so the lower the overall efficiency.

\section{AdAPTATION OF THE LINEAR-Assisted TECHNIQUE FOR VLC}

Taking the block diagram in figure 2 as a reference, the proposed adaptation of the linear-assisted technique for VLC is shown in figure 4 and an example of the light output signals is shown in figure 5. The proposal follows the same principle, but in this case the high efficiency power stage is made of a Class E amplifier instead of a DC-DC converter. The Class E amplifier is being linear-assisted by a linear amplifier.

The idea of adapting the linear-assisted technique for VLC 


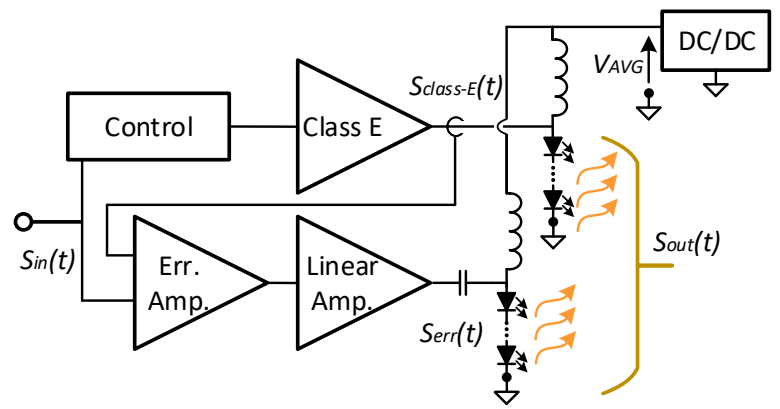

Figure 4: Block diagram of the proposed linear-assisted Class E amplifier using the sum of the light.

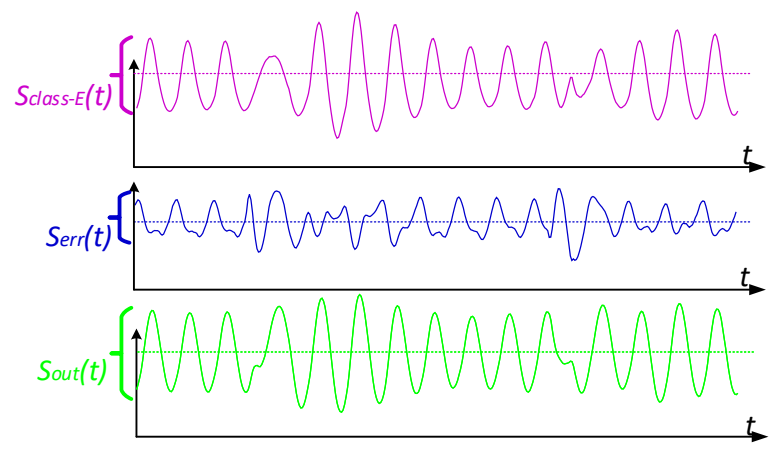

Figure 5: Output light signals in a the linear-assisted circuitry.

lays on taking advantage of the light allowing to sum both signals in light instead of electrically. This modification strongly simplifies the design because, in one hand, the design of the circuitry that combines both signals is not longer necessary. On the other hand, eliminating the electrical connection avoids the influence between the different circuitry involved. This influence is specially critical in resonant circuits such as Class E amplifier, in which the efficiency and correct behavior strongly depends on the correct tuning of the resonant circuit. Figure 4 shows how the linear-assistance is adapted to VLC. Instead of having one string of LEDs, the load is split into two strings and each one is connected to the Class $\mathrm{E}$ amplifier or the linear amplifier respectively. Both strings are biased by an external DC-DC converter, which controls the average current $I_{A V G}$ and the average voltage $V_{A V G}$ across the strings. By controlling the average current, the converter ensures that the LED strings are always working within the linear region regardless the temperature effect. As it can be seen in figure 1 , choosing the biasing point in the middle of the linear region maximizes the amplitude allowed for the communication signal within the linear region. In figure 5, an example of the communication signal and the sum of the light effect is depicted. As it can be seen, each string has an average value that generates a constant light, performing the illumination task of the system. On the other hand, due to the connection of each amplifier to an independent string, each one delivers a variable light, proportional to the signal $s_{\text {class }-E}(t)$ or $s_{\text {err }}(t)$, around the average value mentioned before. The resulting light is the sum of $s_{\text {class }-E}(t)$ and $s_{\text {err }}(t)$, giving $s_{\text {out }}(t)$, and since $s_{\text {err }}(t)$ is the error between the input communication signal $s_{i n}(t)$ and $s_{\text {class }-E}(t)$, by summing the error to $s_{\text {class }-E}(t)$, the result $s_{\text {out }}(t)$ is a closer version of $s_{i n}(t)$.

\section{EXPERIMENTAL RESULTS}

As experimental results, a linear-assisted Class E amplifier is properly designed and build. The circuitry is divided into four parts: the Class $\mathrm{E}$ amplifier, the error amplifier, the linear amplifier and the external DC-DC converter, which is out of the scope of this paper.

The design lays on the idea of summing the signals in light instead of electrically. The Class E amplifier and the linear amplifier are connected to an individual LED string made of 8 LEDs each.

\section{A. Power consumption and efficiency}

The overall power of the system is $8.3 \mathrm{~W}$ divided into biasing and communication signal. The strings are biased using an external DC-DC converter which delivers an average $V_{A V G}$ $=27 \mathrm{~V}$, resulting a $I_{A V G}=0.23 \mathrm{~A}$. The biasing power is around $75 \%$ of the total power. The class $\mathrm{E}$ and the linear amplifier delivers the communication power, which is the remaining $25 \%$ of the overall power. In order to keep a high efficiency, the class $\mathrm{E}$ delivers most of the communication power $(92 \%)$ at a high efficiency $(81 \%)$.

\section{B. Modulation scheme}

In order to validate the communication capability of the proposal, a 16-PSK (Phase-Shift Keying) digital phase modulation is used with a carrier frequency of $1 \mathrm{MHz}$. The modulation has 16 different symbols, encoding 4 bit each, reaching a bit rate of $0.5 \mathrm{Mbps}$. The Class $\mathrm{E}$ amplifier can achieve its maximum performance when the amplitude of the output signal does not vary, and since the 16-PSK is a phase modulation, it does not carry any information on the amplitude of the signal, therefore the amplitude of the signal is kept constant.

\section{Class $E$ amplifier design}

Figure 7 shows the Class E amplifier circuit.

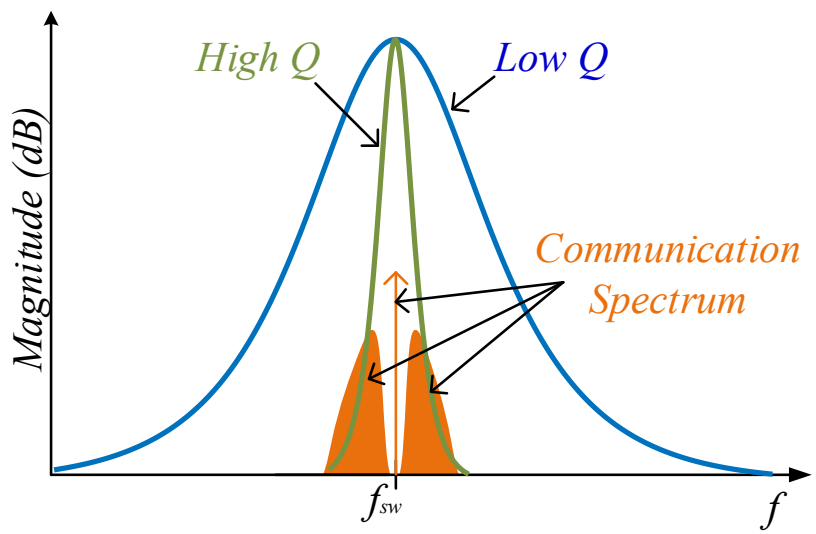

Figure 6: Effect of the $\mathrm{Q}$ of the filter on the communication signal in the Class E amplifier.

The Class E amplifier is designed according to the modulation scheme previously mentioned. The Class E amplifier 


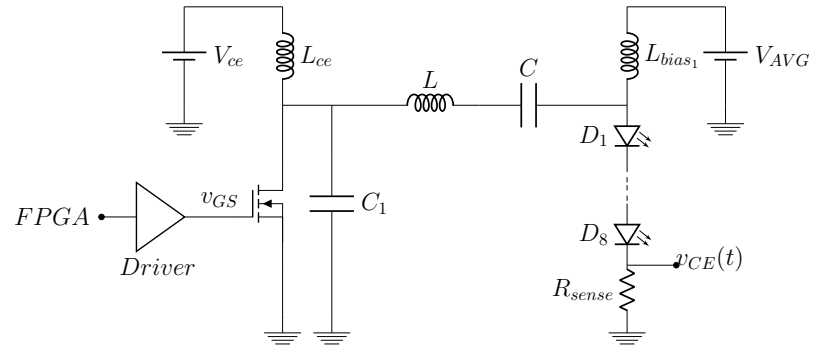

Figure 7: Class E amplifier circuit.

is designed for a switching frequency of $f_{s w}=1 \mathrm{MHz}$, reproducing a sine signal in the output of the same frequency. The amplitude of the output signal is kept constant, so the duty cycle D is fixed to 0.5. The MOSFET used is a PD84010S$E$ RF MOSFET and the driver used is a EL7155 high speed driver. The amplifier is connected to a LED string of 8 XLamp $M X-3$ LEDs. The design of the Class E amplifier lays on the design of the output resonance circuit make up of $C_{1}, C \mathrm{y}$ $L$. The center frequency of the resonant circuit is defined by the switching frequency of $1 \mathrm{MHz}$ and the $\mathrm{Q}$ of the resonant circuit defines the bandwidth of the converter, which depends on the modulation scheme. The effect of the $\mathrm{Q}$ of the filter on the communication signal can be seen in figure 6 . The higher the $\mathrm{Q}$, the lower the bandwidth of the resonant output filter. In order for the Class $\mathrm{E}$ amplifier to be able to reproduce the communication signal, the spectrum of the signal must be within the bandwidth of the output filter. In line with [19], a rough estimation of the bandwidth necessary BW is obtained as follow

$$
B W=\frac{2}{T_{s}}=250 k H z,
$$

where $T_{s}$ is the symbol period. The $\mathrm{Q}$ factor can be calculated by using the definition of the $\mathrm{Q}$ factor

$$
Q=\frac{f_{s w}}{\Delta f_{s w}}=\frac{1 M H z}{250 k H z}=4 .
$$

Table I: VALUES OF THE RESONANT CIRCUIT COMPONENTS FOR THE CLASS E AMPLIFIER.

\begin{tabular}{ccc}
\hline$C_{1}$ & $C$ & $L$ \\
\hline $1.94 n F$ & $3.768 n F$ & $10.5 \mu H$ \\
\hline
\end{tabular}

Due to the low $\mathrm{Q}$ necessary, the traditional design rules for the Class $\mathrm{E}$ amplifier that assume high Q cannot be used. Instead of these rules, by following [20], [21], the values of $C_{1}, C$ y $L$ are obtained and are shown in the table I.

As it can be seen in the figure 7 , a resistance $R_{\text {sense }}$ is placed in series with the LED string to measure the current thought the LED. This current is needed by the error amplifier to generate $s_{e r r}(t)$. Finally, $L_{b i a s_{1}}$ is used as a biasing coil for the LED string.

\section{Linear-assisted circuit design}

Figure 8 shows the circuit of the error amplifier and linear amplifier altogether. The first part generates the error signal $s_{e r r}(t)$ between the Class E amplifier signal $s_{\text {class }-E}(t)$ and the input signal $s_{i n}(t)$.

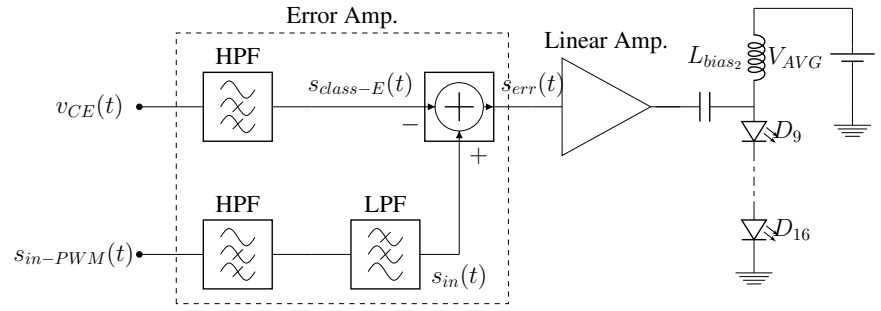

Figure 8: Error amplifier and linear amplifier circuit.

Like in the case of the Class $\mathrm{E}$ amplifier, the linear amplifier is connected to a 8 LED string, and the LED string is externally biased by a DC-DC converter which delivers the $V_{A V G}$ necessary through the coil $L_{b i a s_{2}}$. The linear amplifier delivers the error signal $s_{e r r}(t)$, which is the difference between the desired communication signal $s_{i n}(t)$ (generated by the FPGA) and the communication signal that actually delivers the Class E amplifier $s_{\text {class }-E}(t)$. The Class E communication signal is obtained from the shunt resistance $R_{\text {sense }}$ connected in series with the LED string. The voltage across the resistance is proportional to the current through the LED string, and therefore, it has DC component due to the bias needed. Since the error must be calculated between the communication signal, a $2^{\circ}$ order butterworth High-Pass Filter (HPF) is used to obtain $s_{\text {class }-E}(t)$ without DC component.

The desired communication signal is performed by filtering a PWM signal generated by a FPGA. A HPF eliminates the DC component of the $s_{i n-P W M}(t)$ and a Low-Pass Filter (LPF) that eliminates the PWM components and keeps the communication signal spectrum. The frequency of the $s_{i n-P W M}(t)$ and the cut-off frequencies $f_{c}$ of the filters are in the table II.

$s_{\text {err }}(t)$ is obtained by subtracting $s_{\text {class }-E}(t)$ to $s_{\text {in }}(t)$. $s_{\text {err }}(t)$ is fed directly to the LT1206 linear amplifier. For simplicity, the amplifier is design to have a gain of 1 , so the output is $s_{e r r}(t)$ too. Since the error amplifier is made up of low power operational amplifiers, a linear amplifier is needed in order to provide the power needed for the LEDs.

Table II: DESIGN PARAMETERS OF THE ERROR CALCULATION CIRCUIT.

\begin{tabular}{ccc}
\hline$s_{i n-P W M}$ & LPF $f_{c}$ & HPF $f_{c}$ \\
\hline $30 \mathrm{MHz}$ & $3 \mathrm{MHz}$ & $10 \mathrm{kHz}$ \\
\hline
\end{tabular}

\section{E. Delay calculation}

In order to properly obtain the error signal $s_{\text {err }}(t)$, $s_{\text {class }-E}(t)$ and $s_{i n}(t)$ should have the minimum delay between them. Since the desired signal $s_{i n}(t)$ and the Class E amplifier (through $v_{G S}(t)$ ) are controlled by the FPGA, the synchronization can be implemented also into itself.

In the path from the FPGA to $s_{\text {class }-E}(t)$, the delay of the driver, the Class $\mathrm{E}$ amplifier and the HPF must be taken into account. On the other hand, in the path from the FPGA to 


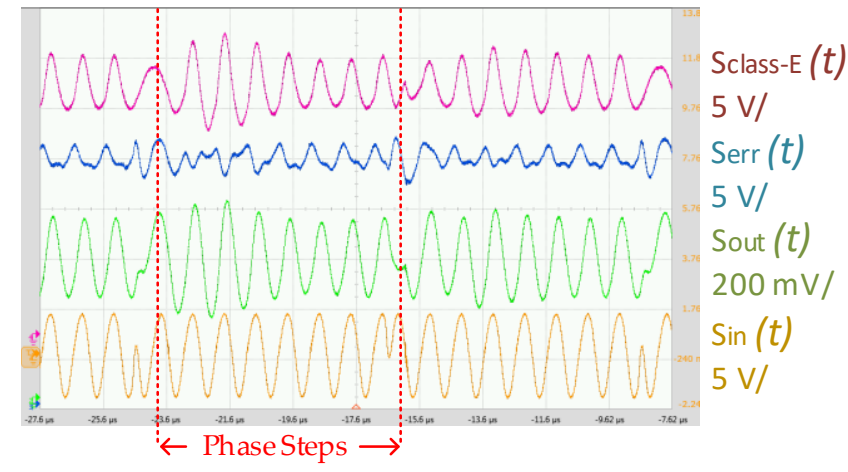

Figure 9: Most representative waveforms of the system during a phase step. $s_{\text {class }-E}(t), s_{\text {err }}(t)$ and $s_{i n}(t)$ are measured in the circuit and $s_{\text {out }}(t)$ is obtenined by measuring the output light.

$s_{i n}(t)$, the delay of the HPF and the LPF must be considered. As it is mentioned before, both HPF are designed equally, so the delay of both are equal.

In the Class $\mathrm{E}$ amplifier path, the driver introduces a delay of $4^{\circ}$ and the Class $\mathrm{E}$ amplifier, acording to [20], the delay is $26^{\circ}$. In the $s_{i n}(t)$ path, the only delay considered is the LPF delay, which is $20^{\circ}$. Acording to this, the PWM that generates the $s_{i n}(t)$ must be delayed $10^{\circ}$ on the FPGA.

\section{F. Working example}

The communication capability is tested by reproducing a 16PSK phase digital modulation with a $1 \mathrm{MHz}$ carrier frequency, achieving a bit rate of $0.5 \mathrm{Mbps}$.

Figure 9 shows the most representative waveforms of the communication system. $s_{i n}(t)$ is the desired communication signal used as a reference and $s_{\text {class }-E}(t)$ is the signal delivered by the Class $\mathrm{E}$ amplifier. The objective is to generate an output signal $s_{\text {out }}(t)$ as close as possible to the reference signal $s_{i n}(t)$. The distortion during a phase step is noticeable in $s_{\text {class }-E}(t)$, and because of this, the error signal $s_{e r r}(t)$ has a higher value during the phase step. The error signal $s_{\text {err }}(t)$ is delivered by the linear amplifier. In order to prove the concept of the sum of the signals in light, $s_{\text {out }}(t)$ is obtained by a optical receiver $P D A 10 A-E C$ placed in front of both strings with a separation up to $1 \mathrm{~m}$. This signal shows a closer version of $s_{i n}(t)$ by adding $s_{\text {class }-E}(t)$ and $s_{\text {err }}(t)$ contributions.

In order to evaluate the communication, the received signal is demodulated with and without the linear-assistance in order to compare the performance. This can be seen on the constellation diagram in Figure 10 after demodulating. The symbols without the linear assistance show a higher dispersion around the ideal symbol, where in contrast, adding the effect of the linear assistance reduces the dispersion of the symbols.

Other way to evaluate the communication is obtaining the error vector $e_{v}$ [22] defined as

$$
e_{v}^{[i]}=v_{r x}^{[i]}-v_{i d}^{[i]},
$$

where $v_{r x}^{[i]}$ is $i^{t h}$ received symbol and $v_{i d}^{[i]}$ is the $i^{t h}$ ideal symbols. In the case of a sequence of $m$ symbols, the Error-

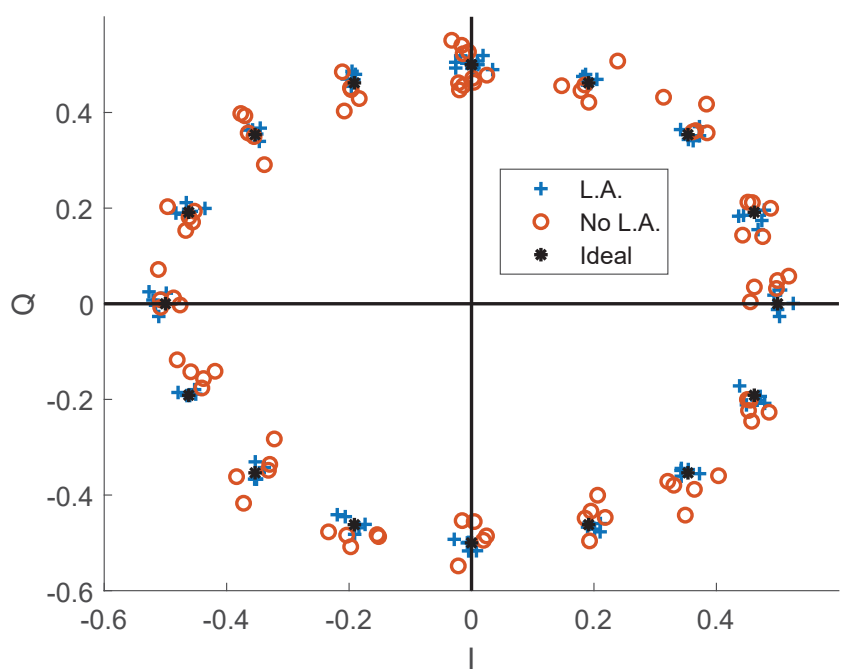

Figure 10: Constellation diagram of the prototype with Linear Assitance (L.A.) and without Linear Assitance (No L.A.)

Vector Magnitude $\left(E V M_{r m s}\right)$ is calculated, which is the normalize root mean square over a sequence of $\mathrm{m}$ symbols as follow

$$
E V M_{r m s}=\sqrt{\frac{\sum_{i=1}^{m}\left|v_{r x}^{[i]}-v_{i d}^{[i]}\right|^{2}}{\sum_{i=1}^{m}\left|v_{i d}^{[i]}\right|^{2}}}
$$

By comparing the $E V M_{r m s}$ with and without linear assistance, the effect of adding it on the communication can be measured.

Table III: RMS VALUE OF THE ERROR VECTOR.

\begin{tabular}{cc}
\hline$e_{v R M S}$ with linear assitance & $e_{v R M S}$ without linear assitance \\
\hline $3.8 \%$ & $8.58 \%$
\end{tabular}

As it is shown in table III, the linear assistance is able to reduce the error significantly, which is in line with the effect on the costelation diagram seen before.

\section{CONCLUSIONS AND FUTURE WORK}

In this paper, a linear-assisted VLC transmitter make of a Class E amplifier and a linear amplifier is presented. The proposal takes advantage of the light and simplifies the linear-assisted technique by avoiding the connection between both amplifiers. In addition, without electrical connection, the design of both amplifiers is independent and there is no interaction between them, which is especially critical when the performance depends on the tune of an output filter (i.e. Class E amplifier). The main advantage of the traditional linear-assisted transmitter is kept, which is the possibility of controlling the distortion of the output communication signal, specially critical in VLC systems. The proposal is able to reproduce a phase digital 16-PSK modulation with a carrier frequency of $1 \mathrm{MHz}$, reaching a bit-rate of $0.5 \mathrm{Mbps}$. Due to 
the fact that the Class $\mathrm{E}$ amplifier delivers most of the power and the linear amplifier stage only delivers the error signal, the proposal achieves a electrical efficiency of $75 \%$ in the signal generation (higher than the alternatives based on solely linear amplifier) and a $85 \%$ overall efficiency considering the signal and the biasing contribution of the DC-DC converter.

\section{REFERENCES}

[1] Cisco Systems. (2017) Cisco visual networking index: Global mobile data traffic forecast update, 2016-2021.

[2] "IEEE standard for local and metropolitan area networks-part 15.7: Short-range wireless optical communication using visible light," IEEE Std 802.15.7-2011, pp. 1-309, Sept 2011.

[3] A. Jovicic, J. Li, and T. Richardson, "Visible light communication: opportunities, challenges and the path to market," IEEE Communications Magazine, vol. 51, no. 12, pp. 26-32, December 2013.

[4] H. Elgala, R. Mesleh, and H. Haas, "Indoor optical wireless communication: potential and state-of-the-art," IEEE Communications Magazine, vol. 49, no. 9, pp. 56-62, September 2011.

[5] D. Karunatilaka, F. Zafar, V. Kalavally, and R. Parthiban, "Led based indoor visible light communications: State of the art," IEEE Communications Surveys Tutorials, vol. 17, no. 3, pp. 1649-1678, thirdquarter 2015.

[6] H. Chun, S. Rajbhandari, G. Faulkner, D. Tsonev, E. Xie, J. J. D. McKendry, E. Gu, M. D. Dawson, D. C. O’Brien, and H. Haas, "LED based wavelength division multiplexed $10 \mathrm{~Gb} / \mathrm{s}$ visible light communications," Journal of Lightwave Technology, vol. 34, no. 13 , pp. 3047-3052, July 2016.

[7] J. Vučić, C. Kottke, K. Habel, and K. D. Langer, " 803 Mbit/s visible light WDM link based on DMT modulation of a single RGB LED luminary," in 2011 Optical Fiber Communication Conference and Exposition and the National Fiber Optic Engineers Conference, March 2011, pp. 1-3.

[8] F.-M. Wu, C.-T. Lin, C.-C. Wei, C.-W. Chen, Z.-Y. Chen, and H.T. Huang, "3.22-Gb/s WDM visible light communication of a single RGB LED employing carrier-less amplitude and phase modulation," in 2013 Optical Fiber Communication Conference and Exposition and the National Fiber Optic Engineers Conference (OFC/NFOEC), March 2013, pp. 1-3.

[9] Y. Wang, L. Tao, X. Huang, J. Shi, and N. Chi, "8-Gb/s RGBY LEDBased WDM VLC system employing high-order CAP modulation and hybrid post equalizer," IEEE Photonics Journal, vol. 7, no. 6, pp. 1-7, Dec 2015 .

[10] J. Rodriguez, D. G. Lamar, P. F. Miaja, and J. Sebastian, "Reproducing single-carrier digital modulation schemes for VLC by controlling the first switching harmonic of the DC-DC power converter output voltage ripple," IEEE Transactions on Power Electronics, vol. 33, no. 9, pp. 7994-8010, Sept 2018.

[11] J. Rodriguez, D. G. Lamar, P. F. Miaja, D. G. Aller, and J. Sebastian, "Power efficient VLC transmitter based on pulse-width modulated DCDC converters and the split of the power," IEEE Transactions on Power Electronics, pp. 1-1, 2018.

[12] J. Sebastian, D. G. Lamar, D. G. Aller, J. Rodriguez, and P. F. Miaja, "On the role of power electronics in visible light communication," IEEE Journal of Emerging and Selected Topics in Power Electronics, pp. 1-1, 2018.

[13] J. Rodriguez, D. G. Lamar, D. G. Aller, P. F. Miaja, and J. Sebastian, "Efficient visible light communication transmitters based on switchingmode DC-DC converters," Sensors, vol. 18, no. 4, 2018.

[14] P. F. Miaja, M. Rodriguez, A. Rodriguez, and J. Sebastian, "A linear assisted DC/DC converter for envelope tracking and envelope elimination and restoration applications," IEEE Transactions on Power Electronics, vol. 27, no. 7, pp. 3302-3309, July 2012.

[15] D. Li, Y. Zhang, M. Rodíguez, and D. Maksimovic, "Band separation in linear-assisted switching power amplifiers for accurate wide-bandwidth envelope tracking," in 2014 IEEE Energy Conversion Congress and Exposition (ECCE), Sep. 2014, pp. 1113-1118.

[16] P. F. Miaja, J. Sebastián, R. Marante, and J. A. García, "A linear assisted switching envelope amplifier for a UHF polar transmitter," IEEE Transactions on Power Electronics, vol. 29, no. 4, pp. 1850-1861, April 2014.
[17] F. H. Raab, P. Asbeck, S. Cripps, P. B. Kenington, Z. B. Popovic, N. Pothecary, J. F. Sevic, and N. O. Sokal, "Power amplifiers and transmitters for RF and microwave," IEEE Transactions on Microwave Theory and Techniques, vol. 50, no. 3, pp. 814-826, March 2002.

[18] L. R. Kahn, "Single-sideband transmission by envelope elimination and restoration," Proceedings of the IRE, vol. 40, no. 7, pp. 803-806, July 1952.

[19] J. Proakis, Digital Communications, ser. Electrical engineering series. McGraw-Hill, 2008

[20] M. Kazimierczuk and K. Puczko, "Exact analysis of Class E tuned power amplifier at any Q and switch duty cycle," IEEE Transactions on Circuits and Systems, vol. 34, no. 2, pp. 149-159, February 1987.

[21] M. K. Kazimierczuk, "Class E tuned power amplifier with nonsinusoidal output voltage," IEEE Journal of Solid-State Circuits, vol. 21, no. 4, pp. 575-581, Aug 1986.

[22] E. McCune, Practical Digital Wireless Signals, U. Cambridge, Ed. Cambridge Univ. Press, 2010. 